\title{
Case Report: Cushings Syndrome Presenting as Hypertensive Emergency with Acute Pulmonary Odema
}

\begin{abstract}
A 22 Year old obese woman presented to emergency medicine department in severe respiratory distress. It was sudden in onset progressive \& associated with cough with pinkish frothy sputum. Her vitals on arrival to ER were BP of $190 / 130 \mathrm{mmHg}$, HR of $136 \mathrm{bpm}$, RR of $34 \mathrm{cpm}, \mathrm{SpO}_{2}$ of $72 \%$ on room air. On examination, there were diffuse fine end inspiratory crepitations bilaterally with severe respiratory distress. Patient was immediately treated for hypertensive emergency with pulmonary odema with intravenous Furosemide, IV Nitroglycerin Oxygen therapy with NIV CPAP.

Upon further evaluation in the observation unit, it was found that the patient was also having hyperglycemia, hypocalcemia hypokalemia, metabolic alkalosis, obesity, purple striae over the abdomen, with irregular menstrual cycles. A screening test of overnight dexamethasone suppression test was done and found serum cortiso to be significantly elevated $(51.49 \mu \mathrm{g} / \mathrm{dL})$, confirming the diagnosis of cushings syndrome presenting with hypertensive emergency and acute pulmonary odema.
\end{abstract}

\section{Background}

Hypertension (HTN) is an important but largely treatable risk factor for cardiovascular disease that affects almost one-third of Americans and approximately 1 billion people worldwide [1,2] Hypertensive emergency a disease state defined by acute TOD (target organ damage), manifest by newly developed clinical sequelae or diagnostic test abnormalities. A hypertensive emergency can exist in patients with or without underlying chronic HTN [3]. This is a rare case of cushings syndrome presenting to emergency with hypertensive crisis and acute pulmonary odema requiring non invasive ventilator support and intravenous antihypertensives for stabilization.

\section{Case Presentation}

A 22 year old obese woman presents to emergency medicine with sudden onset severe respiratory distress associated with cough and pinkish frothy sputum since 2 hours.

A. Vitals on Arrival: $\mathrm{BP}=190 / 130 \mathrm{mmHg}, \mathrm{HR}=136 \mathrm{bpm}, \mathrm{RR}=34$ cpm, $\mathrm{SpO}_{2}=72 \% \mathrm{RA}$.

B. Primary Survey: Airway=severe respiratory distress, diaphoretic, unable to speak full sentences, Breathing=severe tachypnoea, Circulation=bounding pulses, $\mathrm{CRT}<3$ secs.

\section{Critical actions taken}

1. Large bore IV line secured on left forearm.

2. Head end elevation up to 45 degrees

3. Oxygen supplementation with non rebreather mask followed by NIV CPAP $10 \mathrm{cms}$ of $\mathrm{H}_{2} \mathrm{O}$.

\section{Journal of}

Emergency Medicine \& Critical Care

\section{P.V Sukesh*, P.V Sai Satyanarayana, Pailla Naveen Reddy, Ravi N and Rahul Kumar Vojjini}

Department of Emergency Medicine, Kamineni Institute of Medical Sciences, India

Address for Correspondence

P.V Sukesh, Department of Emergency Medicine, Kamineni Institute of Medical Sciences, Narketpally, Nalgonda - 508254 India, Email: Drsukeshpv@gmail.com

Submission: 07 December, 2017

Accepted: 09 April, 2018

Published: 18 April, 2018

Copyright: (๑) 2018 Sukesh PV. et al. This is an open access article distributed under the Creative Commons Attribution License, which permits unrestricted use, distribution, and reproduction in any medium, provided the original work is properly cited.

4. Injection Furosemide $40 \mathrm{mg}$ IV stat (2 doses).

\section{History of presenting illness}

Patient was apparently asymptomatic 2 hours back when she developed shortness of breath which rapidly progressed and was associated with cough and pink frothy sputum. It was also associated with severe sweating and palpitations. There was no history of fever, chest pain, syncopal attacks or fatigue. Patient has a history of irregular menstrual cycles with a denovo diagnosis of hypertension 4 days prior to the arrival to emergency. She was kept on Telmisartan $40 \mathrm{mg}$ by a local practitioner and was not treated for irregular menstrual cycles.

\section{Investigations}

Initial chest $\mathbf{x}$-ray: Homogenous opacities bilaterally with basal clearing. Repeat $\mathrm{x}$-ray after resolution of pulmonary odema was normal without any evidence of mass lesions.

\section{D echo (bedside sonosite)}

Cardiac: Left ventricular hypertrophy with no RWMA normal systolic function and mild to moderate diastolic dysfunction. No valvular abnormalities noted. Pulmonary ultrasonography bedside revealed multiple vertical hyperechoic radial B lines suggestive of pulmonary odema.

Biochemical investigations suggested as:

1. Hypocalcemia: $6.9 \mathrm{mg} / \mathrm{dl}$

2. Hypokalemia: $2.8 \mathrm{mmol} / \mathrm{L}$

3. Hyperglycemia: $294 \mathrm{mg} / \mathrm{dl}$

4. Other initial biochemical parameters were within normal limits.

Haematological investigations were within normal limits apart from mild leucocytosis.

After stabilization an overnight dexamethasone suppression test was performed in view of clinical suspicion of cushings syndrome.

a) $1 \mathrm{mg}$ dexamethasone was given at 11:00 PM in the night. 
b) Early morning serum cortisol sample was sent at 8:00 AM.

c) Serum cortisol was found to be significantly elevated after overnight dexamethasone suppression test. $(51.49 \mu \mathrm{g} / \mathrm{dl})$.

Renal artery Doppler was found to be normal and there were no renal parenchymal changes.

Patients' thyroid profile was within normal limits.

Lower limb pulses were normal bilaterally.

\section{MRI Brain}

Initial plain MRI brain suggested a strong suspicion of pituitary adenoma for which a dynamic MRI brain imaging was planned with contrast. Dynamic imaging though was inconclusive, the pituitary adenoma could not be ruled out and the patient was referred to endocrinologist for further workup.

\section{Differential diagnosis}

Secondary causes of hypertension are important while treating a young patient with hypertension. They include most commonly renal parenchymal and vascular abnormalities, thyroid abnormalities, coarctation of aorta, drugs, pregnancy (pre-eclampsia), endocrine abnormalities. Renal causes (2.5-6\%) of hypertension include renal parenchymal and renal vascular diseases. Renovascular hypertension (RVHT) has become increasingly recognized as an important cause of clinically atypical hypertension and chronic kidney disease [4].

Endocrine abnormalities account for only 1-2\% of cases and include exogenous or endogenous hormonal abnormalities. Exogenous causes include administration of steroids. Endogenous hormonal causes include: Cushings syndrome, Primary hyperaldosteronism, Pheochromocytoma, Congenital adrenal hyperplasia. Cushings syndrome being a rare cause of hypertension can also produce hypertensive emergencies if there is no adequate hemodynamic control.

\section{Treatment}

Patient was treated aggressively in the line of hypertensive emergency with acute pulmonary odema. Intravenous diuretics and antihypertensives were initiated along with oxygen therapy. The oxygen therapy was given with non invasive ventilator support of continuous positive airway pressure of $10 \mathrm{cms}$ of $\mathrm{H}_{2} \mathrm{O}$ while endotracheal intubation and mechanical ventilator support was kept on standby. Potassium and calcium supplementation was given along with metformin $500 \mathrm{mg}$ twice daily for glycemic control. Patient responded well and significantly improved with non invasive ventilation, oxygenation improved and symptoms subsided within 6 hours.

\section{Follow-up}

Patient was advised for endocrinologist follow up for further management and evaluation. Endocrinologist has confirmed the diagnosis of pituitary adenoma causing Cushing's disease and neurosurgical intervention was planned upon further consultation with Neurosurgical team.

\section{Discussion}

Cushing's syndrome reflects a constellation of clinical features that result from chronic exposure to excess glucocorticoids of any etiology. The disorder can be ACTH-dependent (e.g. pituitary corticotrope adenoma, ectopic secretion of ACTH by nonpituitary tumor) or ACTH-independent (e.g. adrenocortical adenoma, adrenocortical carcinoma, nodular adrenal hyperplasia), as well as iatrogenic (e.g. administration of exogenous glucocorticoids to treat various inflammatory conditions). Cushing's syndrome is generally considered a rare disease. It occurs with an incidence of 1-2 per 100,000 populations per year. In the overwhelming majority of patients, Cushing's syndrome is caused by an ACTH-producing corticotrope adenoma of the pituitary, as initially described by Harvey Cushing in 1912. Cushing's disease more frequently affects women, with the exception of prepubertal cases, where it is more common in boys. Only $10 \%$ of patients with Cushing's syndrome have a primary, adrenal cause of their disease (e.g. autonomous cortisol excess independent of ACTH), and most of these patients are women [4].

Glucocorticoids affect almost all cells of the body, and thus signs of cortisol excess impact multiple physiologic systems, with upregulation of gluconeogenesis, lipolysis, and protein catabolism causing the most prominent features. In addition, excess glucocorticoid secretion overcomes the ability of $11 \beta-\mathrm{HSD} 2$ to rapidly inactivate cortisol to cortisone in the kidney, thereby exerting mineralocorticoid actions, manifest as diastolic hypertension, hypokalemia, and edema. Excess glucocorticoids also interfere with central regulatory systems, leading to suppression of gonadotropins with subsequent hypogonadism and amenorrhea. The majority of clinical signs and symptoms observed in Cushing's syndrome are relatively nonspecific and include features such as obesity, diabetes, diastolic hypertension, hirsutism, and depression that are commonly found in patients who do not have Cushing's. Therefore, careful clinical assessment is an important aspect of evaluating suspected cases. A diagnosis of Cushing's should be considered when several clinical features are found in the same patient, in particular when more specific features are found [4].

The most important first step in the management of patients with suspected Cushing's syndrome is to establish the correct diagnosis. Most mistakes in clinical management, leading to unnecessary imaging or surgery, are made because the diagnostic protocol is not followed. This protocol requires establishing the diagnosis of Cushing's beyond doubt prior to employing any tests used for the differential diagnosis of the condition [4]. Cushing's syndrome's cardiovascular complications usually occur due to hypertension, end organ damage such as left ventricular heart failure, diastolic and ischemic myocardial heart failure, which are rather seen in chronic cases of the disease and are often irreversible [5]. Cardiac function in cushings syndrome suggests, impaired left ventricular filling with diastolic hypertension, increased left ventricular mass, with preserved ejection fraction and systolic function. Recently $2 \mathrm{D}$ speckle tracking strain imaging technique has shown a significant improvement in LV structural and functional abnormalities in cushings syndrome following serum cortisol level correction [6].

In the present case, acute respiratory distress with hypertensive crisis was observed. She improved clinically with respect to control of hypertension and acute pulmonary odema. After careful clinical examination and laboratory investigations a diagnosis of 
Citation: Sukesh PV, Sai Satyanarayana PV, Naveen Reddy P, Ravi N, Rahul Kumar V. Case Report: Cushings Syndrome Presenting as Hypertensive Emergency with Acute Pulmonary Odema. J Emerg Med Critical Care 2018;4(1): 3.

cushings syndrome leading to uncontrolled hypertension and acute pulmonary odema was made. Cortisol suppression can be done with ketoconazole or etomidate in nonanesthetic doses. Ketoconazole is used for treatment of cushings syndrome because it inhibits adrenal glucocorticoid synthesis. However, there have been few published studies on its utility in ectopic cushings syndrome, and the results have been controversial [7]. In severe cases of cortisol excess, etomidate can be used to lower cortisol. It is administered by continuous IV infusion in low, nonanesthetic doses [4].

\section{Conclusion}

Cushing syndrome, though itself being a rare condition, the possibility of it leading to hypertensive crisis of secondary origin and causing severe respiratory failure should be emphasized in an emergency unit while treating hypertensive urgency and emergency. The diagnosis of Cushing's disease is important for both emergency physicians and critical care providers because the identification of a secondary cause of hypertension is primary before diagnosing the patient with essential hypertension. Secondary causes of hypertension are treatable and needs further evaluation for the formation of diagnosis. As an emergency physician identification of a secondary cause will lead to an appropriate follow up arrangement for the patient and for critical care providers the identification of the cause can lead to better results and better control of blood pressure especially when the patient presents with a hypertensive emergency (end organ damage).

\section{Take Home Messages}

1. Cushings syndrome is a rare cause of hypertensive emergency to be considered in emergency room.

2. Cardiac manifestations occur in cushings syndrome and might be the first manifestation for that patient.

3. Immediate workup to rule out secondary causes of hypertension in any patient of hypertensive emergency is an important step to be taken in the emergency room itself.

\section{References}

1. Mozaffarian D, Benjamin EJ, Go AS, Arnett DK, Blaha MJ, et al. (2015) Heart disease and stroke statistics-- 2015 update: a report from the American Heart Association. Circulation 131: e29-e322.

2. Lackland DT, Weber MA (2015) Global burden of cardiovascular disease and stroke: hypertension at the core. Can J Cardiol 31: 569-571.

3. Levy PD, Brody A (2018) Hypertension. In: Walls RM, Hockberger RS, Gausche-Hill M, et al. (eds) Rosen's Emergency Medicine: concepts and clinical practice $\left(9^{\text {th }}\right.$ edn $)$, Elsevier, USA, pp. 1007.

4. Arlt W (2015) Disorders of the adrenal cortex. In: Kasper D, Fauci A, Hauser $S$, et al. (eds) Harrison's principles of internal medicine $\left(19^{\text {th }}\right.$ edn $)$, McGrawHill Education, USA.

5. Melmed S, Polonsky K, Larsen PR, Kronenberg H (2011) Williams Textbook of Endocrinology (12 $2^{\text {th }}$ edn), Elsevier, USA, pp. 1-1920.

6. De Leo M, Pivonello R, Auriemma RS, Cozzolino A, Vitale P, et al. (2010) Cardiovascular disease in Cushing's syndrome: heart versus vasculature. Neuroendocrinology 92 Suppl 1: 50-54.

7. Winquist EW, Laskey J, Crump M, Khamsi F, Shepherd FA (1995) Ketoconazole in the management of paraneoplastic Cushing's syndrome secondary to ectopic adrenocorticotropin production. J Clin Oncol 13: 157164. 